\title{
GADOLINIUM-ENHANCED MRI IN THE EVALUATION OF MINIMALLY INVASIVE TREATMENTS OF THE PROSTATE: CORRELATION WITH HISTOPATHOLOGIC FINDINGS
}

\author{
BENJAMIN T. LARSON, JOSEPH M. COLLINS, CHRISTIAN HUIDOBRO, ALBERTO CORICA, \\ SANTIAGO VALLEJO, AND DAVID G. BOSTWICK
}

\begin{abstract}
Objectives. To explore the use of magnetic resonance imaging (MRI) with gadolinium enhancement as a noninvasive method to image the extent of ablation after minimally invasive treatment. Minimally invasive methods for ablating prostatic tissue have emerged as a viable option in the treatment of prostate disease. As these devices enter the mainstream of patient care, imaging methods that verify the exact location, extent, and pattern of the ablation are needed.

Methods. Nineteen patients with prostate cancer were evaluated. All received some type of minimally invasive treatment, post-treatment gadolinium-enhanced MRI sequences, and radical retropubic prostatectomy for histopathologic evaluation. Visual comparisons of gadolinium defects and areas of coagulation necrosis as seen on histopathologic evaluation were made by us. Volumetric and two-dimensional area measurements of the ablation lesions were also compared for correlation between the MRI and histopathologic results.

Results. Gadolinium-enhanced MRI could be matched to histopathologic findings by visual comparison in 17 of the 19 cases. Surgically distorted histopathologic specimens and a small periurethral lesion caused 2 patients to have MRI and histopathologic results that could not be matched. Complete volumetric measurements were available for 16 of the 19 patients and correlated strongly $(r=0.924)$. The two-dimensional area data for all patients also showed significant correlation $(r=0.886)$.

Conclusions. Correlation with histopathologic findings showed gadolinium-enhanced MRI to be useful for determining the location, pattern, and extent of necrosis caused within the prostate by minimally invasive techniques. Gadolinium-enhanced MRI gives the urologist a useful tool to evaluate the effectiveness of new minimally invasive therapies.
\end{abstract}

$\mathrm{N}$ umerous minimally invasive methods for ablating prostatic tissue have emerged in the past decade as legitimate alternatives to surgical treatment. Used mainly for treating benign prostatic hyperplasia and prostate cancer, such methods are now being studied in the treatment of other prostate diseases and other malignancies. ${ }^{1-5}$ Minimally invasive procedures use recent technologic advances in thermal and cryogenic therapy, as well as unique ideas in chemical and enzymatic use.

From Scottsdale, Arizona; Department of Radiology, Mayo Clinic, Scottsdale, Arizona; Department of Urology, University of Chile, Santiago, Chile; Department of Urology, Universidad Nacional de Cuyo, Mendoza, Argentina; Instituto Médico de Urología, Quito, Ecuador; Bostwick Laboratories, Richmond, Virginia
Methods using thermal energy to cause tissue ablation include transurethral microwave thermotherapy, ${ }^{6-8}$ transurethral needle ablation, ${ }^{9-11}$ interstitial laser coagulation, ${ }^{12,13}$ high-intensity focused ultrasonography, ${ }^{14-16}$ and water-induced thermotherapy.1,17 Cryosurgical ablation of the prostate (CSAP) methods use either argon gas or liquid nitrogen to freeze prostatic tissue. ${ }^{3,4,18}$ There is special interest in these minimally invasive techniques, because they are expected to substantially decrease patient recovery time, procedure and post-procedure costs, and morbidity. ${ }^{2}$

As these devices proceed through the developmental phase to clinical use, methods for verifying the location, pattern, and volume of ablation become vital to the correct implementation of minimally invasive procedures. The urologist needs to have objective evidence that the vendor's claims for 
a device are true before treating patients. Human histopathologic results are the best absolute method for ascertaining the extent of ablation within the prostate. However, removal of the prostate for pathologic analysis is not practical in patient care. A noninvasive, prostate-preserving method for determining the extent of ablation will be needed as these devices enter clinical practice. The use of magnetic resonance imaging (MRI) with gadolinium enhancement, as defined by Larson et al., ${ }^{19}$ has been described as a noninvasive method to image the extent of ablation after minimally invasive treatment. MRI demonstrating areas void of gadolinium enhancement are characteristic of no blood flow and indicate necrosis. This study sought to verify the ability of MRI with gadolinium to determine the location, pattern, and volume of ablation caused by minimally invasive devices in the prostate in a noninvasive manner. We compared the gadolinium-enhanced MRI results with the matched histopathologic findings of patients treated with various minimally invasive ablative techniques.

\section{MATERIAL AND METHODS}

A total of 19 patients with prostate cancer were evaluated in this unique study. All had undergone a type of minimally invasive treatment, magnetic resonance imaging with gadolinium enhancement, and radical retropubic prostatectomy during a previous study. The post-treatment MRI scans were compared with the whole mount specimen for each patient. The appropriate institutional review board or ethics committee approved each of the studies in which surgery was performed. MRI scans and histopathologic results were taken from studies using CSAP, two different transurethral microwave thermotherapy devices, and water-induced thermotherapy. The patient data included in this study are the only known data of its kind. The details of each study are given only in summary here because the specific results of each method were not the focus of this study. This study used the patient data available to us to show the correlation of MRI to histopathologic evaluations of minimally invasive procedures and was not meant to be a comparison of the relative effectiveness of the minimally invasive procedures.

\section{Minimally INVASIVe Procedures}

Nineteen men with prostate cancer, who had been previously scheduled to undergo radical prostatectomy as their primary treatment, participated in four separate studies. Five men were treated with the CRYOcare system (Endocare, Irvine, Calif) in a study using only a single probe in each lobe to evaluate the ablative temperature for cryosurgery. ${ }^{18}$ Five men received treatment from the ProstaLund system (ProstaLund, Lund, Sweden) in a study designed to evaluate temperatures and heating patterns. ${ }^{20}$ Five men were treated with the Targis system (Urologix, Minneapolis, Minn) during its early development phase. The treatment consisted of a single 60-minute session. The details of standard Targis system treatments can be found elsewhere. ${ }^{5}$ Four men were treated with the Thermoflex water-induced thermotherapy system (Argomed, Cary, NC). Various temperatures, balloon circumferences, and treatment times were used in that study. MRI sequences were performed 6 to 8 days after treatment in all studies. Rad- ical prostatectomy was performed 2 to 6 weeks after treatment in all studies.

\section{MRI WITH GADOLINIUM SEQUENCES}

In all studies, similar MRI sequences with gadolinium enhancement were performed. Different MRI machines were used, but similar pulse sequences were obtained in each case. Standard $\mathrm{T}_{1}$-weighted and $\mathrm{T}_{2}$-weighted MRI sequences were performed. Also, a fast spoiled gradient recalled echo sequence was performed. This fast $T_{1}$-weighted sequence was obtained in about 30 seconds and was repeated before and after gadolinium enhancement with a dynamic series of images at 1, 2, 3, and 4 minutes. Usually on the third or fourth minute of this sequence, the gadolinium-perfusion defect identifying the areas of necrosis was most conspicuous. Prostatic calculi showed as areas of decreased signal on this sequence as well. Therefore, it was important to have the initial unenhanced sequence as a baseline to compare with the gadolinium-enhanced images. All patients were examined 6 to 8 days after receiving minimally invasive treatment with the MRI sequences described above.

\section{Histopathologic EVALUATION}

All prostates removed by radical retropubic prostatectomy were fixed in $10 \%$ buffered formalin. Serial transverse tissue sections of approximately 5 to $8 \mathrm{~mm}$ thickness were prepared from the prostatic apex to the base. Sections were exposed to graded alcohols and xylene, embedded in paraffin, cut in $5-\mu \mathrm{m}$ slices, stained with hematoxylin-eosin, and examined by light microscopy with prostatic mapping.

\section{Comparison of MRI and Histopathologic RESUlts}

A three-part method was used to compare the results of MRI with gadolinium sequences and the histopathologic evaluation. Transverse MRI scans were visually compared with the corresponding whole mount transverse cuts. Areas of gadolinium defect (no blood flow) were compared with the areas of coagulation necrosis with respect to the size, shape, and distinct characteristics. Volumetric measurements of necrosis were taken from the MRI scans and histopathologic results. The volume of perfusion defect in the prostate was calculated using a modified prolate sphere volume formula (anteroposterior $\times$ transverse $\times$ longitudinal $\times 0.5236$ ). These estimates were compared with the volume of necrosis seen at pathologic evaluation and calculated by the same prolate sphere volume formula. The volume of necrosis was determined by the same formula used to determine the volume of perfusion defect. The third part of the comparison was comparing the two-dimensional areas of similar lesions in the MRI scans versus those measured in the whole mount cuts. In this part, either the area of the largest lesion or another comparable and identifiable lesion was used. Statistical comparisons were made by calculating the correlation coefficient between MRI-derived data and histopathologic-derived data; scatter plots of data are shown in Figures 1 and 2.

\section{RESULTS}

\section{VISUAL COMPARISON}

The relationship of gadolinium defects in MRI scans to areas of necrosis seen on histopathologic evaluation was confirmed with the qualitative analysis conducted by us. Of the 19 patients, 17 presented with gadolinium defects that were remarkably similar to the areas of coagulation necrosis in terms of shape, size, and distinct characteristics. One patient, treated with CSAP, presented with 


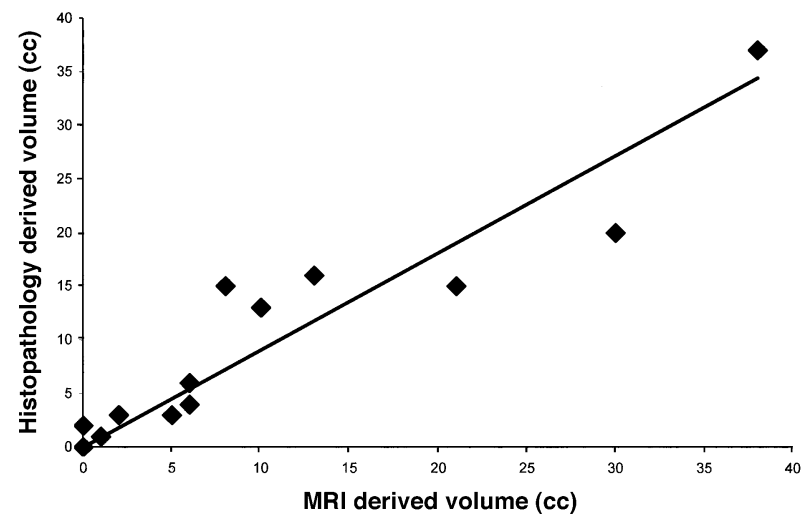

FIGURE 1. Correlation of ablation volumes $(r=$ 0.924). Only 17 of 19 patients had data available for this comparison. Graph also excluded data from one CSAP patient with a surgically distorted histopathologic specimen. MRI-derived volumetric data correlated with histopathologic-derived volumetric data.

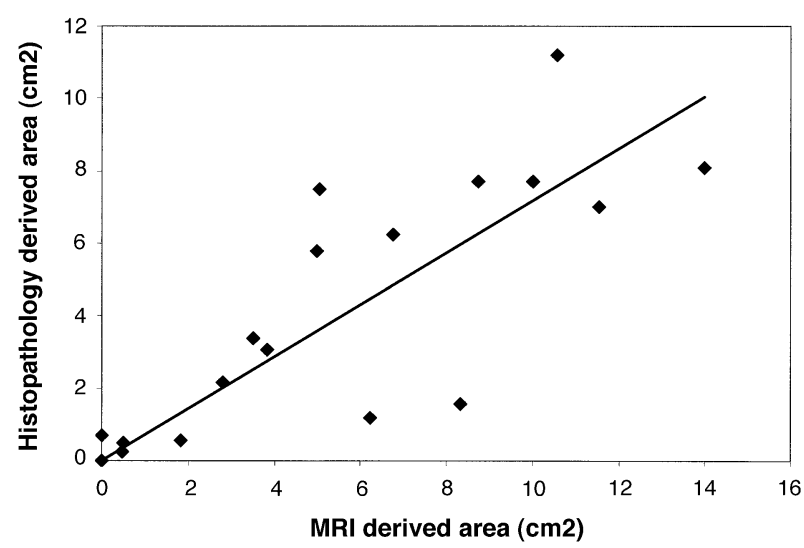

FIGURE 2. Correlation of lesion area $(r=0.886)$. MRIderived areas of two-dimensional lesions correlated with histopathologic-derived data.

gadolinium defects but no similar areas of coagulation necrosis could be seen in the histopathologic evaluation because of the distortion owing to surgical retrieval of the prostate. Another patient, treated with the Targis system, showed a small periurethral lesion in the histopathologic evaluations, but no gadolinium defect was seen in the MRI sequences. Visual comparisons were more accurate with large and complete lesions. Irregular lesions and different angles of imaging and histopathologic cutting made visual comparisons more difficult to assess. As the two examples show (Figs. 3 and 4), the general size and shape of gadolinium defects (Figs. 3A and 4A) represent the areas of necrosis seen during subsequent histopathologic review (Figs. 3B and 4B). Distinct characteristics were identified on both MRI scans and histopathologic slides.

\section{Volumetric Measurements}

Volumetric data for 2 patients treated with the Targis system were unavailable and were not in- cluded in this comparison. One patient treated with CSAP had a distorted histopathologic specimen due to surgery, which created a low volumetric measurement. Including this patient (17 of 19 patients total), the volume of prostate ablation calculated from the perfusion defects seen in the gadolinium-enhanced MRI scans correlated well $(r=$ 0.879 ) with the volumetric measurements of necrosis calculated by histopathologic analysis. Excluding the data from the CSAP patient with a surgically distorted histopathologic specimen, the correlation was even better ( $r=0.924$; Fig. 1).

\section{Two-Dimensional Measurements}

In the comparable two-dimensional areas of all patients, the correlation between areas estimated from the gadolinium perfusion defects and those seen on the histopathologic slides was significant $(r=0.886$; Fig. 2).

\section{COMMENT}

MRI using gadolinium enhancement is becoming a common technique in the study of prostate cancer. ${ }^{21-26}$ Few reports on the use of MRI in prostate evaluations after minimally invasive ablation are available. ${ }^{27}$ Osman et al. ${ }^{28}$ stated that MRI is an accurate noninvasive method to observe the heat pattern distribution within the prostate by linking it directly to the amount of ablation caused. Other studies have reported similar results. ${ }^{19,22,29}$ A recent study used gadolinium-enhanced MRI to image predicted tumor ablation during laser thermal ablation of renal tumors. The correlation coefficient between the ablation seen in gadoliniumenhanced MRI and near real-time $\mathrm{T}_{1}$-weighted thermal mapping was 0.55 , showing a moderate correlation 6 weeks after treatment. Histologic comparisons to gadolinium-enhanced MRI were performed on 1 patient with only minor correlation. However, several months passed between the removal of the tumors and gadolinium-enhanced MRI; the study did conclude that enhancement is the best method for assessing the percentage of viable tumor at present. ${ }^{30}$

The results of this study verify the correlation between MRI with gadolinium enhancement and histopathologic results for determining the amount of necrosis caused within the prostate by minimally invasive techniques. The perfusion defects seen on gadolinium-enhanced MRI scans are an accurate representation of the ablation seen on histopathologic whole mount slides. Volumetric and two-dimensional area measurements also showed strong correlation.

This study gives qualitative validity to the use of MRI with gadolinium enhancement to demonstrate ablation caused by minimally invasive treat- 


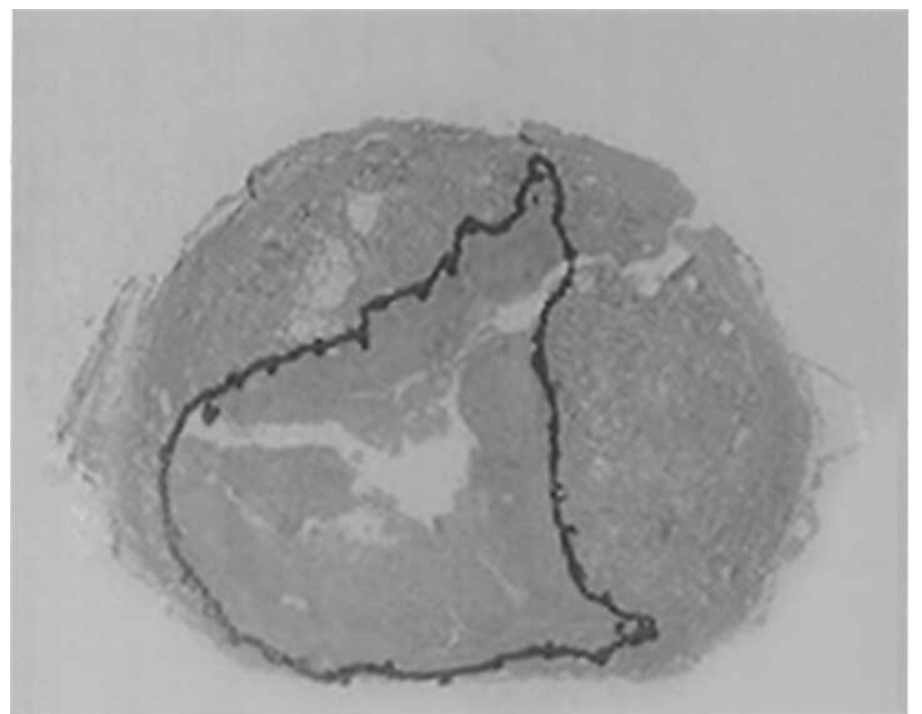

A

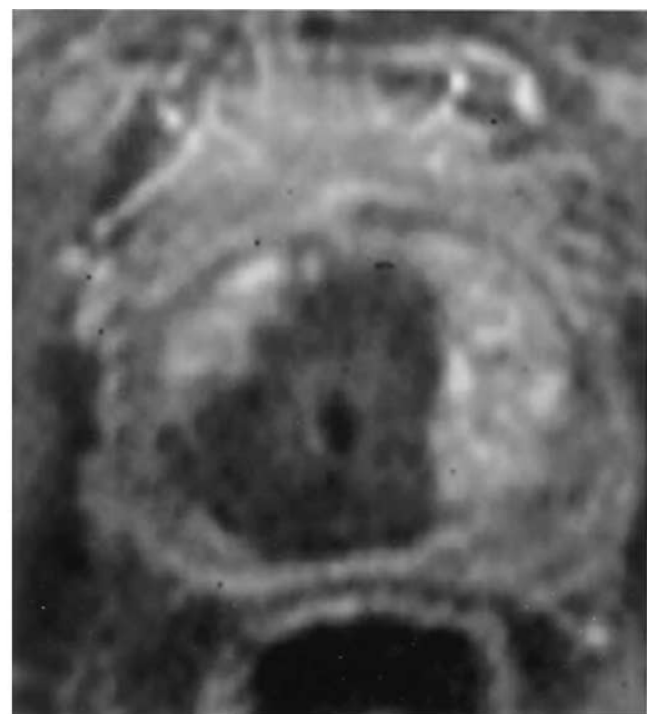

B

FIGURE 3. (A) Histopathologic slide of prostate treated with transurethral microwave thermotherapy; area of coagulation necrosis outlined in black. (B) Gadolinium-enhanced MRI scan; coagulation necrosis seen as dark area void of enhancement.

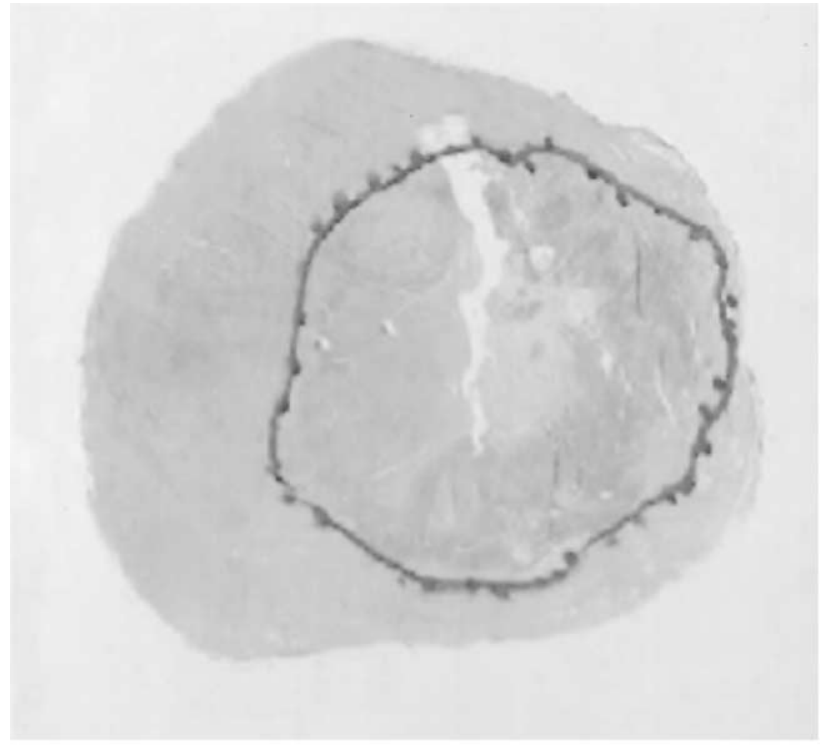

A

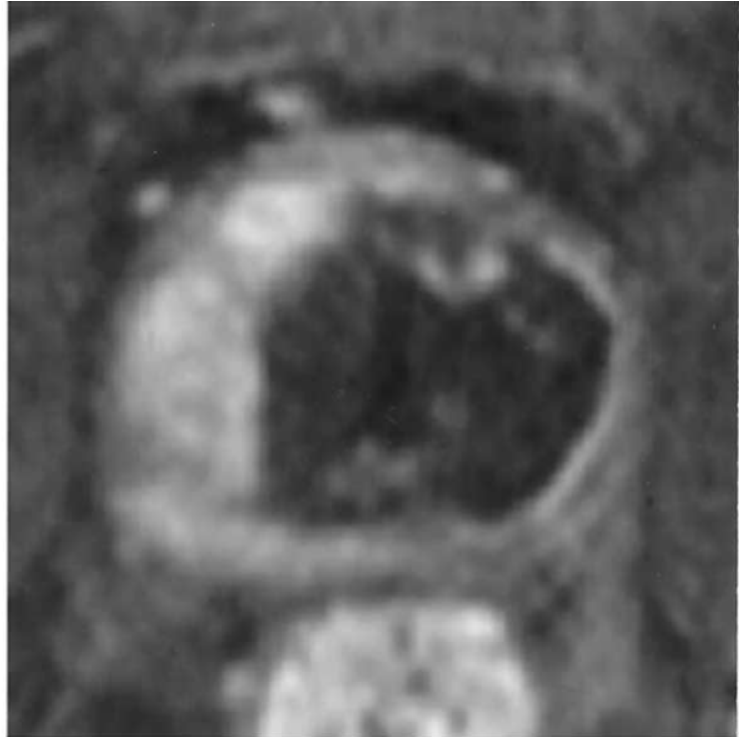

B

FIGURE 4. (A) Histopathologic slide of prostate treated with transurethral microwave thermotherapy; area of coagulation necrosis outlined in black. (B) Gadolinium-enhanced MRI scan; coagulation necrosis seen as dark area void of enhancement.

ments. If MRI is a valid measurement of ablation, it can be used to help understand the actions of the treatments, make fair comparisons between devices, and learn how to use these devices correctly. Some of the discrepancies seen between the MRI data and histopathologic findings can be attributed to the timing of each method. MRI sequences were performed 6 to 8 days after treatment, and swelling and edema would have caused these measurements to be larger. Necrosis near the urethra would be difficult to analyze on histopathologic examination because of tissue sloughing and absorption due to the interval between treatment and removal of the prostate. Difficult surgical removal of the prostate altered the histopathologic data in 1 patient. Slight differences in the slice registration may adversely affect comparison of the MRI scans and the whole mount histopathologic section.

Difficulty arose in the evaluation of CSAP patients because of the damage zone created by cryosurgical treatment. ${ }^{18}$ However, gadolinium defects were not seen in the areas of viable tissue as determined by histopathologic evaluation.

The small number of patients in this study was a 
result of the uncommon nature of the protocols used. Patients who receive post-treatment MRI and undergo subsequent prostatectomy are very few. All such patients were included in this study, and the results show that gadolinium-enhanced MRI can be used to determine the amount, size, and shape of ablation within the prostate caused by minimally invasive devices.

\section{CONCLUSIONS}

The results of this study provide evidence that MRI with gadolinium enhancement is a noninvasive method of verifying the location, pattern, and extent of prostatic tissue ablation produced by minimally invasive methods. Gadolinium-enhanced MRI correlated significantly with whole mount histopathologic sections of the prostate. Gadolinium-enhanced MRI gives the urologist a tool to evaluate the usefulness of new minimally invasive therapies and should be included in subsequent studies. We recommend the use of gadolinium-enhanced MRI in subsequent studies that evaluate the effectiveness and function of new minimally invasive ablative therapies of the prostate.

\section{REFERENCES}

1. Muschter R, Schorsch I, Danielli L, et al: Transurethral water-induced thermotherapy for the treatment of benign prostatic hyperplasia: a prospective multicenter clinical trial. J Urol 164: 1565-1569, 2000.

2. Blute ML, and Larson T: Minimally invasive therapies for benign prostatic hyperplasia. Urology 58: 33-41, 2001.

3. Long JP, Bahn D, Lee F, et al: Five-year retrospective, multi-institutional pooled analysis of cancer-related outcomes after cryosurgical ablation of the prostate. Urology 57: 518523, 2001

4. de la Taille A, Benson MC, Bagiella E, et al: Cryoablation for clinically localized prostate cancer using an argonbased system: complication rates and biochemical recurrence. BJU Int 85: 281-286, 2000.

5. Khair AA, Pacelli A, Iczkowski KA, et al: Does transurethral microwave thermotherapy have a different effect on prostate cancer than on benign or hyperplastic tissue? Urology 54: 67-72, 1999.

6. Brehmer M, and Baba S: Transurethral microwave thermotherapy: how does it work? J Endourol 14: 611-615, 2000.

7. Djavan B, and Marberger M: Minimally invasive procedures as an alternative to medical management for lower urinary tract symptoms of benign prostatic hyperplasia. Curr Opin Urol 11: 1-7, 2001

8. de Wildt MJ, Wagrell L, Larson TR, et al: Clinical results of microwave thermotherapy for benign prostatic hyperplasia. J Endourol 14: 651-656, 2000.

9. Chapple CR, Issa MM, and Woo H: Transurethral needle ablation (TUNA): a critical review of radiofrequency thermal therapy in the management of benign prostatic hyperplasia. Eur Urol 35: 119-128, 1999.

10. Ramon J, Lynch TH, Eardley I, et al: Transurethral needle ablation of the prostate for the treatment of benign prostatic hyperplasia: a collaborative multicentre study. Br J Urol 80: 128-135, 1997.

11. Zlotta AR, Raviv G, Peny MO, et al: Possible mechanisms of action of transurethral needle ablation of the prostate on benign prostatic hyperplasia symptoms: a neurohistochemical study. J Urol 157: 894-899, 1997.

12. Kursh E: Interstitial laser coagulation technique: ILC blueprint. World J Urol 18(suppl 1): S6-S13, 2000.

13. Muschter R, and Whitfield H: Interstitial laser therapy of benign prostatic hyperplasia. Eur Urol 35: 147-154, 1999.

14. Madersbacher S, Kratzik C, and Marberger M: Prostatic tissue ablation by transrectal high intensity focused ultrasound: histological impact and clinical application. Ultrason Sonochem 4: 175-179, 1997.

15. Madersbacher S, Schatzl G, Djavan B, et al: Long-term outcome of transrectal high-intensity focused ultrasound therapy for benign prostatic hyperplasia. Eur Urol 37: 687694, 2000.

16. Hegarty NJ, and Fitzpatrick JM: High intensity focused ultrasound in benign prostatic hyperplasia. Eur J Ultrasound 9: 55-60, 1999.

17. Corica FA, Cheng L, Ramnani D, et al: Transurethral hot-water balloon thermoablation for benign prostatic hyperplasia: patient tolerance and pathologic findings. Urology 56 : $76-81,2000$.

18. Larson TR, Robertson DW, Corica A, et al: In vivo interstitial temperature mapping of the human prostate during cryosurgery with correlation to histopathologic outcomes. Urology 55: 547-552, 2000.

19. Larson TCJ, Bostwick D, and deGetter P: MRIs "GAD defect" can quantify coagulation necrosis in thermotherapy: a new comparative model. J Urol 157: 437, 1997.

20. Schelin S: Microwave thermotherapy in patients with benign prostatic hyperplasia and chronic urinary retention. Eur Urol 39: 400-404, 2001.

21. Adusumilli S, and Pretorius ES: Magnetic resonance imaging of prostate cancer. Semin Urol Oncol 20: 192-210, 2002.

22. D'Angelo MFHK, King BF, Patterson DE, et al: Prospective comparative analysis of MRI gadolinium enhanced central zone perfusion defects using the Prostatron 2.0 and the Targis thermotherapy devices (abstract). J Urol 159(suppl): 304, 1998.

23. Engelbrecht MR, Jager GJ, Laheij RJ, et al: Local staging of prostate cancer using magnetic resonance imaging: a metaanalysis. Eur Radiol 12: 2294-2302, 2002.

24. Gossmann A, Okuhata Y, Shames DM, et al: Prostate cancer tumor grade differentiation with dynamic contrast-enhanced MR imaging in the rat: comparison of macromolecular and small-molecular contrast media-preliminary experience. Radiology 213: 265-272, 1999.

25. Kuhn M, Huttmann P, Spielhaupter E, et al: Clinical value of native and contrast enhanced MRI in staging prostatic carcinoma before planned radical prostatectomy. Rofo Fortschr Geb Rontgenstr Neuen Bildgeb Verfahr 173: 595$600,2001$.

26. Ogura K, Maekawa S, Okubo K, et al: Dynamic endorectal magnetic resonance imaging for local staging and detection of neurovascular bundle involvement of prostate cancer: correlation with histopathologic results. Urology 57: 721-726, 2001.

27. Rouviere O, Lyonnet D, Raudrant A, et al: MRI appearance of prostate following transrectal HIFU ablation of localized cancer. Eur Urol 40: 265-274, 2001.

28. Osman YM, Larson TR, El-Diasty T, et al: Correlation between central zone perfusion defects on gadolinium-enhanced MRI and intraprostatic temperatures during transurethral microwave thermotherapy. J Endourol 14: 761-766, 2000.

29. Larson TR, Collins J, and Bostwick D: MRI can qualify coagulative necrosis in thermotherapy (abstract). J Endourol 1: S111, 1996.

30. Dick EA, Joarder R, De Jode MG, et al: Magnetic resonance imaging-guided laser thermal ablation of renal tumours. BJU Int 90: 814-822, 2002. 